\title{
A General Computational Form for a Class of Nonlinear Systems Incorporating both Spectral and Finite Difference Approximations
}

\author{
By \\ F. Baer and R.L. King
}

Department of Atmospheric Science

Colorado State University

Fort Collins, Colorado

Technical report to the National Science Foundation

Grant No. GA-761

February, 1967
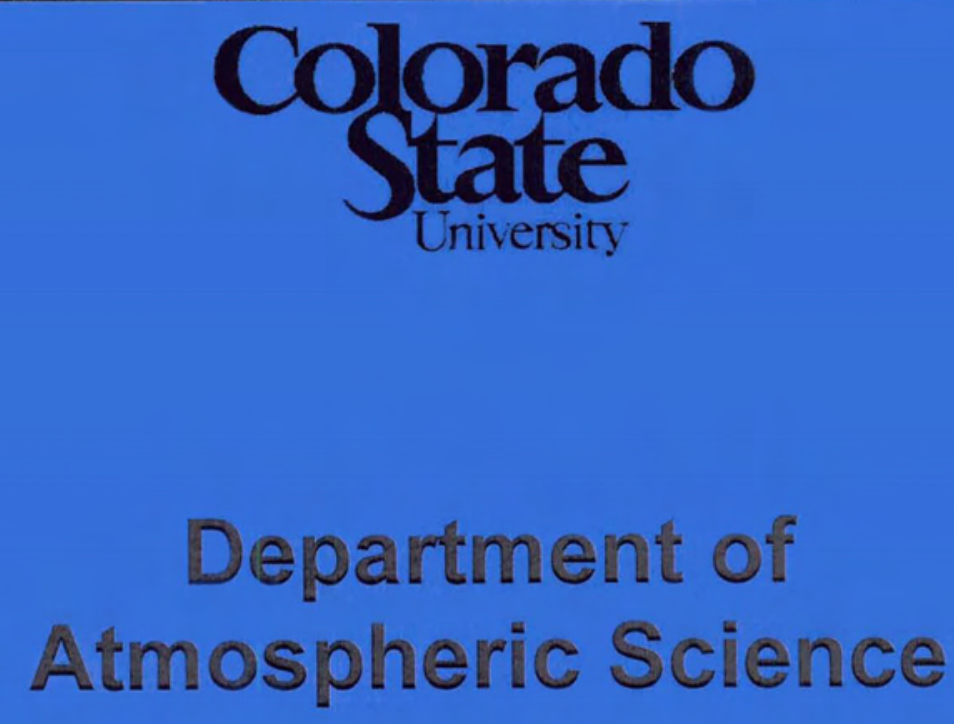

Paper No. 108 
A GENERAL COMPUTATIONAL FORM FOR A CLASS

OF NONLINEAR SYSTEMS INCORPORATING BOTH SPECTRAL

AND FINITE DIFFERENCE APPROXIMATIONS

by

F. Baer and R. L. King

Technical Report to

The National Science Foundation

Grant No. GA-76I

\footnotetext{
Department of Atmospheric Science

Colorado State University

Fort Collins, Colorado

February 1967

Atmospheric Science Paper No. 108
} 
CONTENTS

Page

Abstract

I. Introduction.............. . . . . .

2. Representational similarities of space and spectral domain methods... . 5

3. Reduction of terms involving the time operator ............ . 10

4. Reduction of nonlinear terms...... 13

5. Example 1: Finite difference method. . 23

6. Example 2: Spectral method....... 29

7. Calculation procedure . . . . . . . 35

8. Conclusion... . . . . . . . . . 39

References............. . . . 4 4 
A General Computational Form for a Class

of Nonl inear Systems Incorporating Both Spectral

and Finite Difference Approximations

by

F. Baer and R. L. King

Colorado State University

ABSTRACT

It is shown that a general class of nonlinear partial differential equations--including those frequently used in predicting atmospheric motions--can be converted to computational form by either the "finite-difference" or "spectral" method to yield formally identical equations. These computational equations are then reduced to matrix notation and the nonlinearity (of arbitrary order) is shown to be expressible as a bilinear form. Finally, a method is described whereby these bilinear forms--quadratic forms in the case of even nonlinearity--may be readily programmed and calculated on a digital computer. 
1. Introduction

Traditionally, information available about the behavior of a physical system which could be represented by a set of differential equations was deduced from the linear form of such equations. Those systems in which nonlinearity predominated were largely ignored, primarily because no analytical tools were available for their solution. The development of the digital computer with its large memory and high-speed calculation capability was instrumental in directing attention to nonlinear problems; this instrument has the capacity for solving nonlinear systems numerically.

with reference to the problem of weather prediction, the basic mathematical equations which represent atmospheric flow are fundamentally nonlinear. Thus, this science lay dormant until computing technology advanced sufficiently to stimulate research and experimentation. A classic example of the frustration which inadequate computation facility could cause is vividly described in the work of Richardson (1922). The promise which modern computing technology offers is evident from the fact that weather forecasts are currently being supplied by numerical solution of mathematical representations in many weather centrals around the world; and this development has taken place in less than two decades.

A considerable amount of research effort has been expended to bring the state of numerical forecasting to its present point; a large but unknown amount must yet be employed to extend our knowledge and capability. Such effort should be directed toward a better understanding of the physical aspects of the problem; wherever possible, mathematical aids should be available to the investigator so as not to deter or distract him from his search for better physical insight. It is the purpose of this paper to present such an aid.

Once a set of differential equations is established to describe a physical system, a method of representation 
must be selected to define the values of the dependent variables as a finite set of numbers*. Two such methods are currently applied to weather prediction systems. One-the more popular--involves the transformation of the differntial equations to finite difference equations and is applied to a finite set of points in the space under consideration (space domain). The method was employed in one of the first calculations of numerical weather prediction by Charney, et al. (1950); is being used in research investi-gations of the general circulation of the atmosphere by Leith (1965), Mintz (1965), and Smagorinsky, et al. (1965): and is the method utilized in routine daily weather prediction by the Numerical Weather Prediction unit of ESSA.

The other method--frequently referred to as the spectral domain method--involves the expansion of the dependent variables in a finite series of space-dependent functions (known) and time-dependent coefficients (unknown). Space derivatives are then evaluated by operation on the known functions and the entire system is integrated over the specified domain. The original set of differential equations is thus reduced to a finite set of differential equations in the time-dependent coefficients only. This set of coefficients corresponds (from the computation point of $v i e w$ ) to the time-dependent set of point values established by the first proposed method. The spectral approach has been employed in weather prediction models by Silberman (1954), Bryan (1959), Baer (1961, 1964), and others.

It is not the purpose of this discussion to argue the relative merits of these methods, a question which has already been considered by Ellsaesser (1966). Rather, it is the writers' purpose to establish a general representation and computation scheme which is applicable to either method.

* Clearly this is necessary because the computer requires a finite time for any one calculation; thus it would require an infinite time to establish the continuous variation of any variable. 
In all the numerical models which have been reported, the authors have devoted substantial effort to representing their equations for computation; it is our hope that in the future this effort will be obviated.

At though emphasis has been placed on systems of equations representing atmospheric flow--reflecting the writers' backgrounds--a representation will be presented which covers a broad class of differential equations. Needless to say, any physical system which can be described by these equations may benefit from the representation.

Consider now a system involving the set of $N$ dependent variables $\Phi_{j}$,

$$
\Phi_{j}=\Phi_{j}(\bar{r},+) \quad 1 \leq j \leq N
$$

wherein the dependent variables are related by $N$ differntial equations in both time $(t)$ and space $(\bar{r}$ - the positive vector). Let us further assume that the nonlinearity of the system involves only space-dependent differential operations on the dependent variables. When the dependent variables exist linearly, they may be operated on by both time- and space-dependent operators. We thus consider the general class of $N$ equations,

$$
\begin{aligned}
& \sum_{j=1}^{N} R_{k, j}\left(\Phi_{j}\right)=Z_{k}\left(\Phi_{1}, \cdots, \Phi_{N}\right) ; 1 \leq k \leq N \\
& \left.Z_{k} \equiv \sum_{m=1}^{M} \sum_{m} \prod_{i=1}^{m} F_{0}^{m}, m, i, k \Phi_{i}\right)
\end{aligned}
$$

Here $R_{k, j}$ is an operator in both time and space, whereas $F_{0}^{m}, m, i, k$ is an operator in space alone. The parameters $\mu, s$ and $\sum_{\Gamma_{m}}$ are defined as follows: 


$$
\begin{aligned}
& s_{p} \equiv \sum_{i=1}^{p} s_{i} \\
& \mu_{p}^{m} \equiv\left(s_{p+1}, s_{p+2}, \cdots, s_{m}\right) \\
& \sum_{m} \equiv s_{1}^{N}=1 s_{2}^{N-s_{1}} \cdots s_{m}^{N-s_{m}-1}
\end{aligned}
$$

We note that $\mu_{0}^{m}$ is a vector describing the dependent variables $\Phi_{s_{i}}$ which are involved in any nonlinear product; since each operator $F$ operates only on one dependent variable $\Phi_{s_{i}}$, the index $i$ serves to isolate that variable from the vector $\mu_{0}^{m}$. The order of the nonlinearity is determined by the value of $\mathrm{m}$. Thus (1.1) allows nonlinearity to order $M$ - If the same set of variables as specified by $\mu_{0}^{m}$ are involved in more than one product, a summation over another index would be needed to include all such terms; i.e., we would need $F_{0}^{1}{ }_{0}^{m}, m, i, k, F_{0}^{2}, m, i, k$, etc. This possibility offers no added complication, and we consequently make no symbolic reference to it. Subsequent examples will exemplify this situation. Should a system yield terms with nonlinear products which are not integral, an expansion would have to be performed to make the terms conform to the representation of (1.1).

It is the purpose of the subsequent discussion to show that the system (1.1) can be converted either by spectral expansion or by finite difference means to a computational representation which can be easily applied to digital computing procedures, provided an assumption in the numerical time integration is made. Only space truncation will be considered here. Several examples with meteorological emphasis will be developed to exhibit the utility of the method. 
2. Representational similarities of space and spectral domain methods

We shall show in this section that subject to definition, equation (1.1) can be put into a representation which is identical for both the finite difference and spectral truncation methods. Let us first, however, simplify the time and space operator $R_{k, j}$. Discounting transcendental operators, one may generally write,

$$
R_{k, j}=\sum_{i=1}^{I} T_{k, j, i} G_{k, j, i}
$$

where $T_{k, j, i}$ is an operator in time alone and $G_{k, j, i}$ is an operator in space only. If we introduce (2.1) into (1.1), we have the $N$ equations

$$
\sum_{i}^{I} \sum_{j=1}^{N} T_{k, j, i} G_{k, j, i}\left(\Phi_{j}\right)=Z_{k}\left(\Phi_{1}, \cdots, \Phi_{N}\right)
$$

where $Z_{k}$ is defined as in $(1.1)$.

Finite-difference method: To solve equation (2.2) numerically, one must assign values to the dependent variables, $\Phi_{j}$, at discrete points in space. Customarily, the domain of interest is broken up into a three-dimensional lattice and the variables are assumed known at the intersection points of the lattice. If one numbers these points, the association of this number with any dependent variable specifies the variable's value at that point. It is not essential that the different variables $\Phi_{j}$ be known on the same grid; i.e., a different grid may be assigned to each variable. Thus we shall define the point number for any $\Phi_{j}$ as $\gamma_{j}$ where,

$$
1 \leq \gamma_{j} \leq n_{j}
$$


and the value of the function $\Phi_{j}$ (in principle continuously distributed in space) is given by the set of numbers $\left(\phi j, \gamma_{j}\right) ;$ this set is, of course, time dependent.

We see that by breaking the continuous function into a discrete set, we also generate $n_{k}$ equations in the timedependent quantities $\phi_{k}, \gamma_{k}$ from each of the $k$ differential equations (2.2), resulting in $\sum_{j=1} n_{j}$ equations in the same number of variables $\phi_{j, \gamma_{j}}$. We shall for future reference define

$$
L \equiv \sum_{j=1}^{N} n_{j}
$$

These equations cannot be written explicitly in terms of the variables $\phi_{j, \gamma_{j}}$ until a finite-difference operator is substituted for each space differential operator. The finite-difference operator may depend on all the grid values of the variable as follows:

$$
\left[G_{k, j, i}\left(\Phi_{j}\right)\right]_{\gamma_{k}}=\sum_{\alpha_{j}=1}^{n_{j}} a_{k, j, \alpha_{j}, \gamma_{k}, i \phi_{j, \alpha_{j}} .}
$$

The coefficients "a" in the above equation will be determined from the choice of the finite-difference procedure to be applied. The more common of such operators involve only the near neighbor points to the point in question; only those "a's" for which $\alpha_{j}$ represented the near neighbor points of $\gamma_{k}$ would be nonzero. We shall see subsequently from an example how the fields of "a" are selected. Equation (2.4) is applicable to the left-hand side of (2.2); a similar development is used in the function $Z_{k}$ of $(1.1)$. If

$$
\left.\left[F_{\mu_{0}}^{m}, m, i, k^{(\Phi} s_{i}\right)\right]_{\gamma_{k}}={ }_{\alpha_{s_{i}}^{i}=1}^{\sum_{s}}\left(a_{\mu}^{m}, m, i, k, \alpha_{s_{i}}^{i}, \gamma_{k}\right) \phi_{s}, \alpha_{s_{i}}^{i}
$$


where the finite-difference operator may or may not be the same as in equation (2.4), * we may combine the non I inear terms to yield

$$
\left.\prod_{i=1}^{m} F_{\mu}^{m}, m, i, k^{(\Phi} s_{i}\right)=\sum_{\lambda{ }_{0}^{m}}\left(\prod_{i=1}^{m} \phi_{s_{i}}, \alpha_{s_{i}}^{i}\right) I_{\lambda}^{m}, \mu{ }_{0}^{m}\left(k, \gamma_{k}\right)
$$

with

$$
I_{\lambda{ }_{0}, \mu_{0}^{m}}\left(k, \gamma_{k}\right) \equiv \prod_{i=1}^{m} a_{\mu}^{m}, m, i, k, \alpha_{s}^{i}, \gamma_{k}
$$

Rather than writing out the product of sums, a single sum has been written to be taken over all variations of the vector $\lambda_{0}^{m}=\left(\alpha_{s_{1}}^{1}, \alpha_{s_{2}}^{2}, \alpha_{s_{3}}^{3}, \cdots, \alpha_{s_{m}}^{m}\right)$ where the range of each of the $\alpha^{\prime} s$ is given as $1 \leq \alpha_{s_{i}} \leq n_{s_{i}}$. This is clear from the substitution of (2.5) into the product on the left-hand side of $(2.6)$. The vector $\mu_{0}^{m}$ has been defined previously in $(1.2)$.

Spectral method: In this method it is not necessary to establish discrete values of the dependent variables; one assumes the space dependence to be given by known polynomials in space and each variable is expanded in a series of timedependent coefficients multiplied by the known polynomials. For computation purposes, the series must be finite, and thus truncation is introduced. If for each variable $\Phi_{j}$ there are $n_{j}$ coefficients (the truncation point), we may write

$$
\Phi_{j}=\sum_{\alpha_{j}=1}^{n_{j}} \phi_{j, \alpha_{j}}(+) P_{\alpha_{j}}(\bar{r})
$$

where $P_{\alpha_{j}}$ are the space varying polynomials (known) and the $\phi_{j, \alpha_{j}}$ are the coefficients (time dependent) of the

* If the finite-difference operators applied to the same equation differ for different times, serious computational stability problems may occur. This is not within the domain of our discussion here. 
series. Note that the $\phi_{j, \alpha}$ of this discussion are not the same physical quantities as those of the finite-difference discussion although symbolically they are identical.

It would be desirable to select the polynomials $P$ as the characteristic function of the linearized form of the differential equation under consideration ( 1.1 ), but such functions may not often be available. Furthermore, it would be desirable, but not essential, that the polynomials be orthogonal on integration over the domain of interest. On substitution of the expansion (2.8) into (2.2), we still have $N$ differential equations in both time and space, but we have increased the number of dependent variables to $L$ (see 2.3). The number of equations may be increased to the number of dependent variables and the space dependence may also be removed if each of the $k$ equations is multiplied successively by $P_{\gamma_{k}}$ and integrated over the space domain, where $\gamma_{k}$ goes over its entire range, $1 \leq \gamma_{k} \leq n_{k}$. Thus each of the original equations yields $n_{k}$ equations which are time dependent only.

Consider the space operation on the left-hand side of (2.2). Using the expansion (2.8),

$$
G_{k, j, i}\left(\Phi_{j}\right)=\sum_{\alpha_{j} \phi_{j, \alpha_{j}}}(+) G_{k, j, i}\left(P_{\alpha_{j}}\right) .
$$

Multiplying by $\mathrm{P}_{\gamma_{k}}^{*}$ and integrating (the asterisk denoting the complex conjugate),

$$
\int G_{k, j, i}\left(\Phi_{j}\right) P_{\gamma_{k}^{*}} d \sigma=\sum_{\alpha_{j}} a_{k, j, \alpha_{j}}, \gamma_{k}, i \phi_{j, \alpha_{j}}
$$

where

$$
a_{k, j, \alpha_{j}, \gamma_{k}, i} \equiv \int G_{k, j, i}\left(P_{\alpha_{j}}\right) \cdot P_{\gamma_{k}^{*}} d \sigma
$$


A similar procedure may be applied to the function $Z_{k}$ of $(1.1)$. We substitute (2.8), multiply by $P_{\gamma_{k}^{*}}^{*}$ and integrate to yield

$$
\left.\prod_{i=1}^{m} F_{\mu_{0}^{m}}^{m}, m, i, k^{(\Phi} s_{i}\right)=\sum_{\lambda_{0}^{m}}\left(\prod_{i=1}^{m} \phi_{s_{i}}, \alpha_{s_{i}}^{i}\right) I_{\lambda_{0}}^{m}, \mu_{0}^{m}\left(k, \gamma_{k}\right)
$$

where

$$
I_{\lambda} m, \mu_{0}^{m}, k, \gamma_{k}=\delta \prod_{i=1}^{m} F_{\mu_{0}}^{m}, m, i, k{ }_{\alpha_{s}}{ }^{m} P_{\gamma_{k}}^{*} d \sigma
$$

The vectors $\lambda_{0}^{m}$ and $\mu_{0}^{m}$ have the same meaning as those used in (2.6) although the range of the indices $\alpha_{s_{i}}^{i}$ must be established from the series given in (2.8).

We are now in a position to convert (2.2) into a set of equations which are only time dependent in the variables $\phi_{j, \alpha_{j}}$. For the finite-difference method we substitute (2.4) on the left-hand side and (2.6) on the right-hand side; for the spectral method we first multiply (2.2) by $P_{\gamma}^{*}$ and integrate, then substitute (2.9) on the left-hand sidekand (2.11) on the right. Either of these procedures leads to the following equation:

$$
\begin{aligned}
& \sum_{i=1}^{N} \sum_{j}^{n_{j}} T_{k} T_{k, j, i}\left(\phi_{j, \alpha}\right) a_{k, j, \alpha_{j}, \gamma_{k}, i}= \\
& \sum_{m=1}^{M}\left[\sum_{m}^{\sum_{m}} \sum_{m}\left(\prod_{i=1}^{m} \phi_{s_{i}, \alpha_{s}}\right) I_{\lambda_{i}}^{m}, \mu_{0}^{m}\left(k, \gamma_{k}\right)\right] .
\end{aligned}
$$

Thus we see that the computational form of the general differential equation (2.2) is representationally identical for both the finite-difference and spectral methods. This similarity allows for the development of a general computation 
scheme applicable to either method. It is only necessary to note the definitions of the dependent variables $\phi_{j, \alpha_{j}}(t)$ and the constant coefficients "a" and "I" as defined by $(2.4,2.7)$ or $(2.10,2.12)$ respectively for the finite-difference or spectral methods.

3. Reduction of terms involving the time operator

We consider now a simpler representation of the spatial truncatio: of our differential equations which is applicable to either the finite-difference or spectral method. We have,

$$
\sum_{i} \sum_{j} \sum_{j} T_{k, j, i}\left(\phi_{j, \alpha}\right) a_{k, j, \alpha_{j}, \gamma_{k}, i}=B_{k, \gamma_{k}}
$$

where $B$ is defined as the right-hand side of (2.13).

Let us now define a column vector $\Phi_{j}$ which includes all the $\alpha_{j}$ elements of $\phi$ for a given $j$; these may be either the point values of the variables or the expansion coefficients and will be written

$$
\Phi_{j} \equiv\left\{\phi_{j, \alpha_{j}}\right\}
$$

with dimensions $\left(n_{j} \times 1\right)$. A composite vector, including all the vectors of the different dependent variables will be defined as $\chi$ and written

$$
X \equiv\{\Phi\}
$$

of dimensions $(L \times 1)$.

Consider now the following matrices involving the time

$$
\begin{aligned}
& \text { operators } T_{k, j, i} \text {; }
\end{aligned}
$$

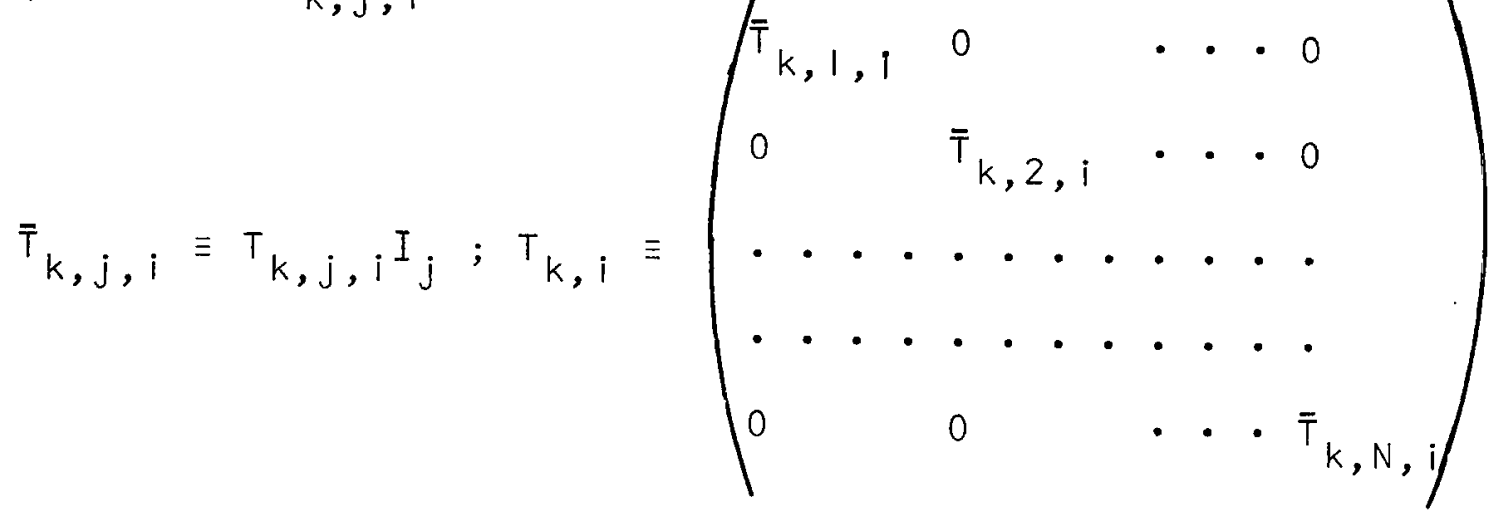


Here the matrices $I_{j}$ are unit matrices of dimensions $\left(n_{j} \times n_{j}\right)$ and consequently the $\bar{T}_{k, j, i}$ are of the same dimensions. Since the matrices $T_{k, i}$ are generated as diagonal matrices with $\bar{T}_{k, j, i}$ on the diagonal, their dimensions are $(L \times L)$.

Finally, let us define a column vector of elements $a_{k, j, \alpha}, \gamma_{k}, i$ in which the elements are listed varying most rapidly on $\alpha_{j}$ and least rapidly on $j$. This vector will be written as

$$
A_{k, \gamma_{k}, i} \equiv\left\{a_{k, i, \alpha_{j}, \gamma_{k}, i}\right\}=\left(\begin{array}{c}
a_{k, 1,1, \gamma_{k}, i} \\
a_{k, 1,2, \gamma_{k}, i} \\
\vdots \\
a_{k, 1, n_{1}, \gamma_{k}, i} \\
a_{k, 2,1, \gamma_{k}, i} \\
\vdots \\
a_{k, N, n_{N}, \gamma_{k}, i}
\end{array}\right)
$$

Clearly the vector has dimensions $(L \times 1)$.

If we introduce the definitions (3.2), (3.3), (3.4), and (3.5) into (3.1), we have the form,

$$
\sum_{i} \tilde{A}_{k, \gamma_{k}, i} T_{k, j} x=B_{k, \gamma_{k}}
$$

where the tilde represents matrix transposition. The elements of "A" are known either from (2.4) or (2.10), and therefore the matrix "A" may be combined with the time operator matrix $T_{k, i}$ to yield a modified matrix. Furthermore, since $x$ does not depend on the index $i$, the operator matrices may be summed over this index to yield

$$
T_{k, \gamma_{k}} \equiv \Sigma_{i} \tilde{A}_{k, \gamma_{k}, i} T_{k, i}
$$

\footnotetext{
a time operator matrix of dimension $(I \times L)$. If we now write a vector which has as elements $T_{k, \gamma_{k}}$ over all $k, \gamma_{k}$, varying $\gamma_{k}$ most rapidly, and write a similar vector made up of the elements $B_{k, \gamma_{k}}$ as follows:
} 


$$
T \equiv\left\{T_{k, \gamma_{k}}\right\} ; B \equiv\left\{B_{k, \gamma_{k}}\right\} \text {, }
$$

$T$ having dimensions $(L \times L)$ and $B$ having dimensions $(L \times 1)$, ( 3.1$)$ is finally reduced to the matrix equation

$$
T X=B(X) \text {. }
$$

Since the function $B$ is generally a non-l inear function of the dependent variable $x$, an analytic solution for $x$ as a function of time usually can not be found. We therefore resort to a numerical solution of (3.9) in time. If we break the time axis into increments of $\Delta t$ and assume that initial values are available at some time $t_{0}$ (consider $t_{0}=0$ for simplicity), we may write a finite-difference equation to replace (3.9) at any time $t=\rho \Delta t$. Let us select an explicit extrapolation scheme (Richtmyer, 1957), although this is not essential. We shall assume that the time operator $T$ at any time $\rho \Delta t$ will predict $x[(\rho+1) \Delta t]$ from all the previous values $\chi(j \Delta t)(j \leq \rho)$ which have been calculated and are known. Thus,

$$
(T X)^{\rho \Delta t}=\sum_{i=0}^{\rho+1} \tau, \rho, i x(i \Delta t)
$$

where the matrices $\tau_{\rho, i}$ are functions of the matrices $a_{k, \gamma_{k}, i}(E q .3 .7)$ and $\Delta t$ (the time truncation interval) and the method of truncation employed. Because we have selected an explicit method, the right-hand side of (3.9) will be evaluated at $\rho \Delta t$ in terms of known quantities. On the assumption that $\tau_{p, p+1}$ is non-singular, we may substitute (3.10) into (3.9) and solve for $x[(p+1) \Delta t]$, the unknown. The resulting finite-difference extrapolation equation becomes,

$$
\begin{gathered}
x[(\rho+1) \Delta t]=-\sum_{i=0}^{\rho} \tau^{-1} \rho, \rho+1^{\tau} \rho, i x(i \Delta t)+ \\
+\tau_{\rho, \rho+1}^{-1} B[x(\rho \Delta t)] .
\end{gathered}
$$


The first term on the right-hand side is easily calculated since it is linear in $x$ and all the $x$ 's are known up to $p \Delta t$. The second term involves the nonlinear products of $x$ at $\rho \Delta t$. To establish the value of each element of the vector $x$ at $o \Delta t$, we see that it will depend upon all the values of the dependent variables $\phi_{j, \alpha}$ for all time increments computed in a linear sense, plus a linear combination of all the nonlinear forms at $\rho \Delta t$. We thus have the general form,

$$
\phi_{k, \gamma_{k}}[(\rho+1) \Delta t]=\sum_{i=0}^{\rho} \sum_{j} \sum_{\alpha_{j}} U_{\rho, j, \alpha_{j}, k, \gamma_{k}, i} \phi_{j, \alpha_{j}}(i \Delta t)+
$$

$$
+\sum_{j} \sum_{\alpha_{j}} v_{\rho, j, \alpha_{j}}, k, \gamma_{k}{ }^{B}, \alpha_{j}[x(\rho \Delta t)] .
$$

where "U" and " $v$ " can be determined from the matrices of $(3.11)$.

It is important to note that the dependent variable can at any time be determined (not including linear terms) by a I inear combination of nonlinear products--the functions ${ }^{B}{ }_{k, \gamma_{k}}$-calculated at the preceding time step. We proceed now to simplify the representation of the functions $B_{k, \gamma_{k}}$.

\section{Reduction of nonlinear terms}

We have seen from (3.12) that the value of each scalar dependent variable $\phi_{j, \alpha}$ can be computed by a linear combination of the nonlinear products " $B$ " as defined by the right-hand side of $(2.13)$ and written

$$
B_{k, \gamma_{k}} \equiv B\left(k, \gamma_{k}\right)=\sum_{m=1}^{M}\left[\sum_{m} \sum_{m}\left(\prod_{i=1}^{m} \phi_{i}, \alpha s_{i}^{i}\right) I_{\lambda}{ }_{0}^{m}, \mu_{0}^{m}\left(k, \gamma_{k}\right)\right] .
$$


We note that for $m=1$ the terms are linear in the $\phi_{j, \alpha_{j}}$ and are easily calculated. We shall thus direct the following discussion to terms with $m>1$. For notational simplicity, we shall suppress the indices $k, \gamma_{k}$, although it shall be understood that the I's of (4.1) depend on them.

Before proceeding with this development, let us establish some definitions which will prove useful in the sequel. Consider the vector $x$ as defined in (3.3). The matrix $\bar{x}$ will be defined as a matrix with zero off diagonal elements (in dimensions of $x$ ) and the vector $x$ as diagonal elements. The dimensions of each element of this matrix will be the dimensions of $x$ and the dimensions of the matrix in terms of its elements will be square and of order $L$, the number of scaler elements in $x$. Thus,

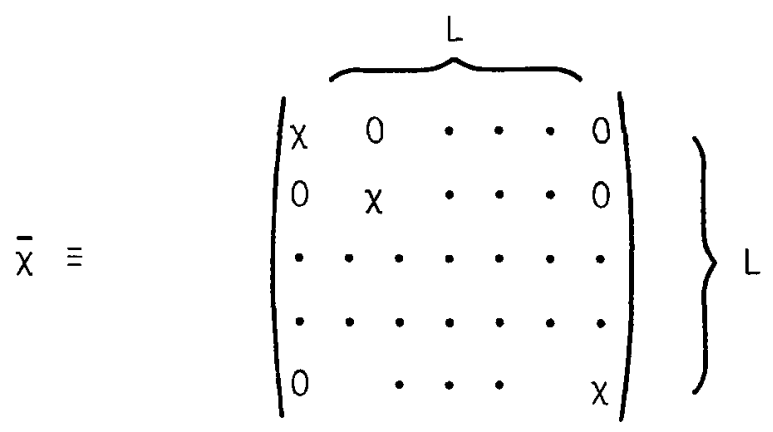

and

$$
\left.\overline{\bar{x}}=\left|\begin{array}{cccccc}
\overline{\bar{x}} & 0 & \cdot & \cdot & \cdot & 0 \\
0 & \bar{x} & \cdot & \cdot & \cdot & 0 \\
\cdot & \cdot & \cdot & \cdot & \cdot & \cdot \\
\cdot & \cdot & \cdot & \cdot & \cdot & \cdot \\
0 & \cdot & \cdot & \cdot & \bar{x}
\end{array}\right|\right\} L
$$

etc. Since $x$ is a vector of order $(L \times 1), \bar{x}$ will be of order $\left(L^{2} \times L\right)$ in the scaler elements $\phi_{j, \alpha_{j}}$. The bar operator as defined by (4.2) may be applied to a vector (say $x$ ) 
as many times as necessary, and we shall abbreviate this multiple operation by the notation,

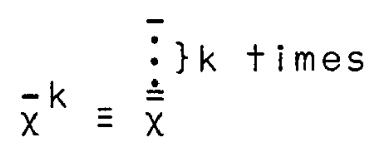

Clearly, $\bar{x}^{-k}$ has dimensions $\left(L^{k+1} \times L^{k}\right)$.

The matrices developed by sucessively increasing the bar operation on $x$ may be multiplied; such a product will be defined for convenience as

$$
\begin{aligned}
& U_{m} \equiv \prod_{k=1}^{m} \bar{x}^{m-k} \\
& U_{0} \equiv 1
\end{aligned}
$$

Care must be taken to perform the multiplication subject to consistency rules for matrix multiplication. However, the multiplication may be performed in reverse if the matrices are transposed. This may be seen from the following two identities:

$$
\begin{aligned}
\tilde{\bar{x}} & =\overline{\tilde{x}} \\
\overline{\bar{x} x} & =\overline{\bar{x}} \bar{x}
\end{aligned}
$$

Both identities are apparent from the definition of the bar operator (4.2a) and the definition for transposition of matrices. Eqs. (4.5) and (4.6) may easily be extended for an arbitrary number of bar operations.

Finally, let us define vectors with dimensions identical to $\Phi_{j}$ and $x$ but with unit elements;

$$
k_{j} \equiv\left\{\begin{array}{c}
1 \\
\vdots \\
\vdots
\end{array}\right\} n_{j} ; \quad k \equiv\left\{\begin{array}{c}
k_{1} \\
k_{2} \\
\vdots \\
k_{N}
\end{array}\right\} \quad L
$$


Quadratic products: We consider now a part of the nonlinear product (4.1) which involves only the quadratic product of the dependent variables $\phi_{j, \alpha_{j}}$ which we shall denote as

$$
Q_{2} \equiv \sum_{S_{1}} s_{2} \lambda_{0}^{2}{ }_{0}^{\phi} s_{1}, \alpha \frac{1}{s_{1}} \phi_{s_{2}}, \alpha_{s_{2}}^{2} I_{\lambda}{ }_{0}^{m}, \mu{ }_{0}^{m}\left(k, \gamma_{k}\right) .
$$

Note from the definition of $\lambda$ following (2.7) that $\lambda_{0}^{2}=\left(\alpha_{s_{1}}^{1}, \alpha_{s_{2}}^{2}\right)$ as used in $(4.8)$. For given dependent variables represented by $s_{1}$ and $s_{2}$, we may sum over all elements $\phi_{s_{i}}, a_{s_{i}}^{i}$ for the quadratic product to get the matrix representation

$$
Q_{2}=\sum_{S_{1} S_{2}} \tilde{\Phi}_{S_{1}}\left(I_{\lambda} m, H_{0}^{m}\right) \Phi s_{2}
$$

Here $\Phi_{s_{1}}$ and $\Phi_{s_{2}}$ are vectors defined by $(3.2)$ and I as used in (4.9) is a matrix made up of the elements I given in $(4.8)$ as

$$
I_{\lambda}{ }_{2}^{m}, \mu_{0}^{m} \equiv\left\{I_{\lambda}^{m}, \mu_{0}^{m}\right\}
$$

with dimensions $\left(n_{s_{1}} \times n_{s_{2}}\right)$. Since the interaction matrix defined in (4.10) is independent of $\alpha_{s_{1}}^{1}$ and $\alpha_{s_{2}}^{2}$, we assign the subscript on $\lambda$ to indicate the number of indices by which $\lambda_{0}^{m}$ has been reduced; i.e.,

$$
\lambda_{2}^{m}=\left(\alpha_{s_{3}}^{3}, \alpha_{s_{4}}^{4}, \cdots, \alpha_{s_{m}}^{m}\right)
$$

We now develop a matrix of dimensions ( $L \times L$ ) which is made up of the sub-matrices defined in (4.10). Since this matrix will have elements depending on the values of $\left(s_{1}, s_{2}\right)$, we may assume that for each product of (4.9) only the element corresponding to $\left(s_{1}, s_{2}\right)$ will be nonzero. Thus, 


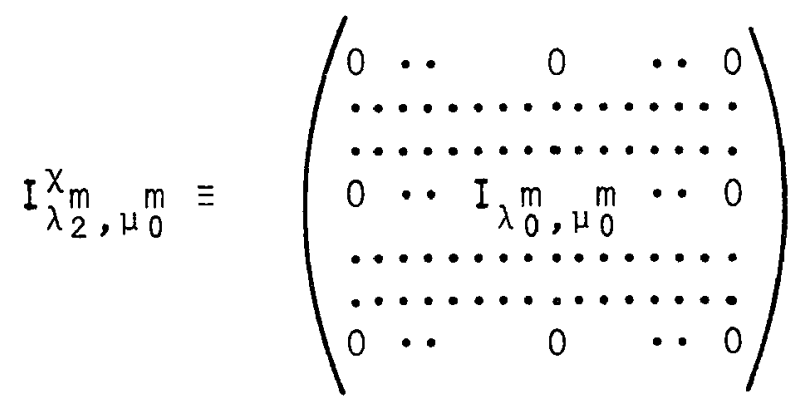

Because al! elements except $\left(s_{1}, s_{2}\right)$ in $(4.11)$ are zero, we may replace the appropriate $\Phi_{s_{i}}$ with $x$ (defined by $E q$. 3.3) in (4.9) and using (4.11) arrive at the equation

$$
Q_{2}=\sum_{S_{1} S_{2}} \tilde{X}\left(I_{\lambda} X_{2}, \mu_{0}^{m}\right) \chi
$$

Finally, since $x$ is independent of $\left(s_{1}, s_{2}\right)$, the summation may be made over all matrices of the type (4.11) to yield the complete matrix

$$
I_{\lambda}{ }_{2}^{m}, \mu_{2}^{m} \equiv \frac{1}{2} \sum_{S_{1} S_{2}}\left[I_{\lambda}^{X_{2}}, \mu_{0}^{m}+\tilde{I}_{\lambda_{2}^{m}, \mu_{0}^{m}}^{m}\right] .
$$

Note that since the matrix $I_{\lambda} m_{2}^{m}, \mu_{2}^{m}$ is no longer dependent on $\left(s_{1}, s_{2}\right)$, the subscript of $\mu$ has been incremented to indicate the number of degrees of freedom by which $\mu_{0}^{m}$ has been reduced. From the definition of $\mu$ in (1.2),

$$
\mu_{2}^{m} \equiv\left(s_{3}, s_{4}, \cdots, s_{m}\right)
$$

This matrix has also been made symmetric to avoid confusion, an operation which may be justified by noting in (4.12) that $Q_{2}$ is a scalar. By introducing (4.13) into (4.12), we get the matrix form of $Q_{2}$,

$$
Q_{2}=\tilde{x}\left(I_{\lambda}{ }_{2}^{m}, \mu_{2}^{m}\right) x
$$


Cubic products: We may express a cubic product of (4.1) by multiplying $Q_{2}$ by another dependent variable (denoted by $s_{3}$ ) and taking the appropriate sums. This yields

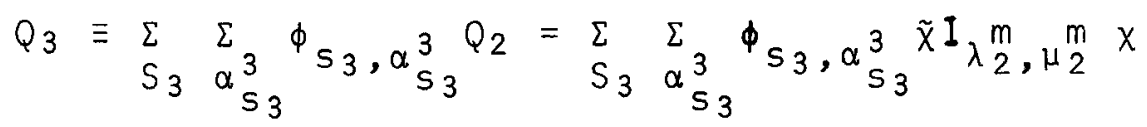

Unlike the quadratic case, this equation depends on only one dependent variable, $s_{3}$. However, we may simulate the method applied to the quadratic case by generating a square matrix of dimensions ( $n_{s_{3}} \times n_{s_{3}}$ ) with only diagonal elements and defined as

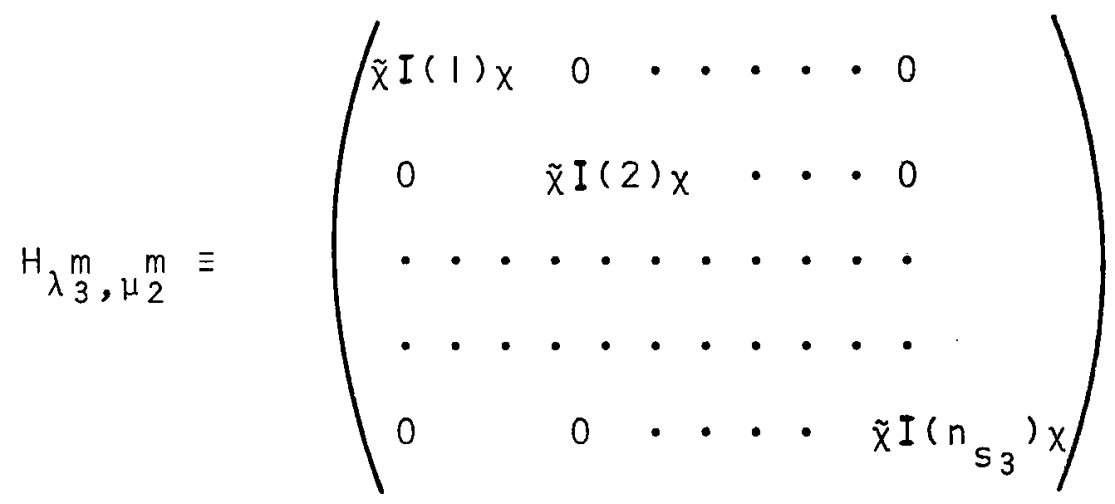

$(4.16)$

with $\quad \lambda_{3}^{m}=\left(\alpha_{s_{4}}^{4}, \alpha_{s_{5}}^{5}, \cdots, \alpha_{s_{m}}^{m}\right)$ and $I(j) \equiv I_{\lambda} m_{2}, \mu_{2}^{m}\left(\alpha_{s_{3}}^{3}=j\right)$.

Substituting $(4.16)$ into $(4.15)$ and introducing the vector with unit elements defined by $(4.7),(4.15)$ becomes

$$
Q_{3}=\sum_{S_{3}} \tilde{K}_{s_{3}}\left(H_{\lambda{ }_{3}, \mu}^{m}\right) \Phi_{2} s_{3}
$$

The subsequent development follows by analogy to (4.11) and (4.13). Develop a square matrix in the elements $H$ (defined by 4.16) with only one non-zero element, a diagonal element at the location $\left(s_{3}, s_{3}\right)$ and with dimensions $(L \times L)$ in the scalars $Q_{2}$; 


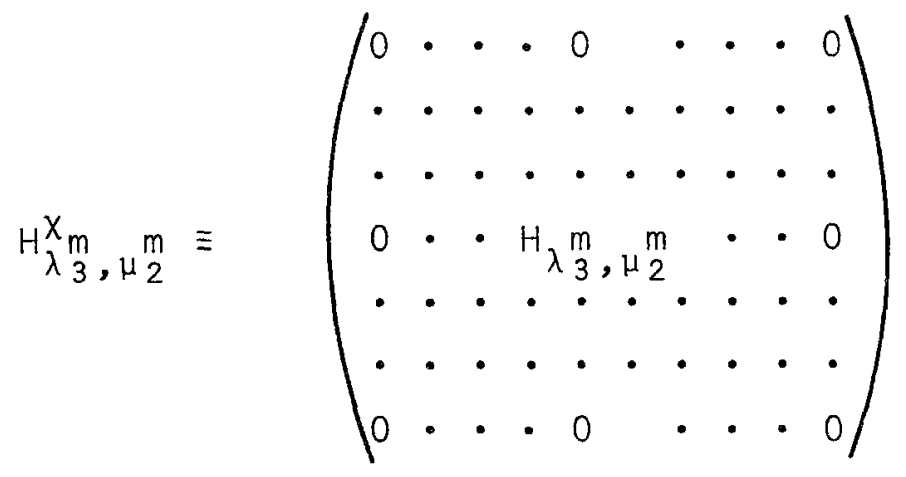

Substitution of $(4.18)$ into $(4.17)$ allows the use of the vector $K(E q .4 .7)$ and $x$ to yield

$$
Q_{3}=\tilde{K}\left(\sum_{S_{3}} H_{\lambda}^{X_{m}}, \mu_{2}^{m}\right) x
$$

The summation of matrices implied in (4.19) leads to a square matrix of order $(L \times L)$ with only diagonal elements, the terms $Q_{2}$ developed for quadratic products; each of these elements is premultiplied by $\tilde{x}$ and postmultiplied by $x$. By using the definition of the bar operator (Eq. 4.2a), we may thus write this sum

$$
\left.\sum_{S_{3}} H_{\lambda_{3}, \mu_{2}}^{X_{m}}=\overline{\tilde{x}}\left(I_{\lambda_{3}, \mu}^{m}\right)_{3}^{m}\right) \bar{x}
$$

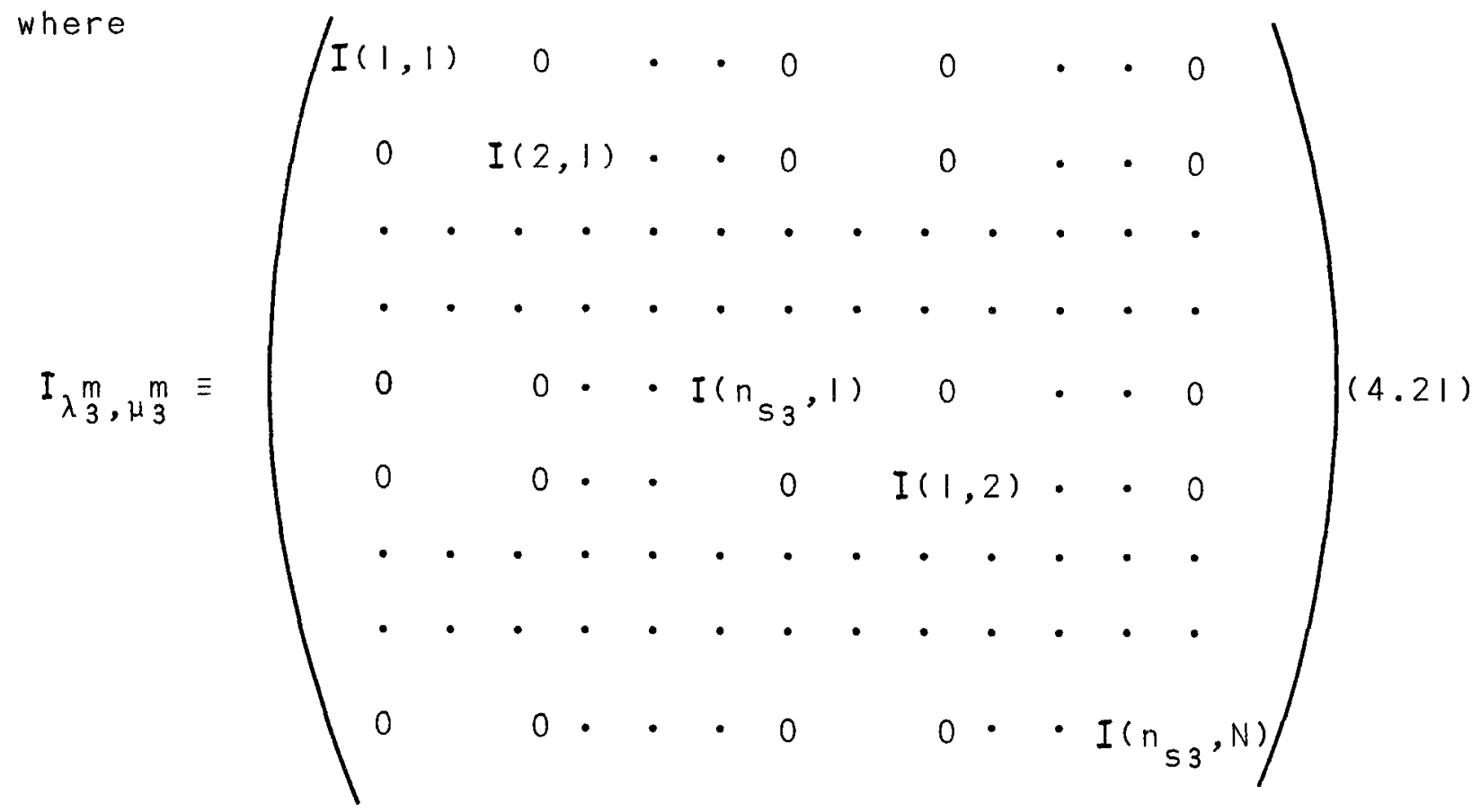


Here $\mu_{3}^{m}=\left(s_{4}, s_{5}, \cdots, s_{m}\right)$ and $I(j, k) \equiv I_{\lambda} m m_{2}^{m}\left(\alpha_{s_{3}}^{3}=j, s_{3}=k\right)$. Note that $I_{\lambda_{3}, \mu_{3}}$ is symmetric since it is made up only of matrices on its diagonal which are themselves symmetric, and has dimensions $\left(L^{2} \times L^{2}\right)$. Utilization of (4.20) together with (4.19) leads to the final form for the cubic product,

$$
Q_{3}=\tilde{K} \overline{\tilde{x}}\left(I_{\lambda} m_{3}, \mu_{3}^{m}\right) \bar{x} x
$$

Quartic products: The quartic product may be expressed from (4.1) by using $Q_{2}$ (Eq. 4.14) and two extra dependent variables,

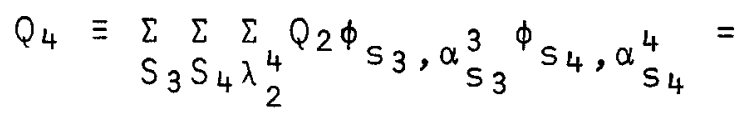

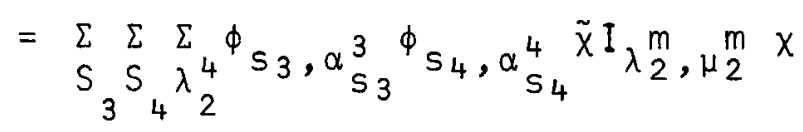

where $\lambda_{2}^{4}=\left(\alpha_{s_{3}}^{3}, \alpha_{s_{4}}^{4}\right)$. The development now follows identically the development for the quadratic case with $Q_{2}\left(\lambda_{2}^{m}, \mu_{2}^{m}\right)$ replacing $I_{\lambda \lambda_{0}^{m}, \mu_{0}^{m}}$. We first establish a matrix similar to (4.10),

$$
I_{\lambda_{4}, \mu_{2}^{m}}^{m}=\left\{\tilde{x} I_{\lambda 2}^{m}, \mu_{2}^{m} x\right\}
$$

with dimensions $\left(n_{s_{3}} \times n_{s_{4}}\right)$. Next we expand on these matrices to create--similar to $(4.11)$--an augmented matrix of dimension $N \times N$ in the $I_{\lambda 4}^{m}, \mu_{2}^{m}$ defined as $I_{\lambda} X_{m}, \mu_{2}^{m}$ with only one non-zero element in location $\left(s_{3}, s_{4}\right)$. Noting from $(4.12)$ that $\Phi_{s_{3}}, \Phi_{s_{4}}$ may be replaced by $x$ in $(4.23)$, the summation over $s_{3}, s_{4}$ may be applied to this newly created matrix and it may furthermore be made symmetric in a fashion similar to (4.13). We now observe that each element of this 
matrix is premultiplied by $\tilde{x}$ and postmultiplied by $x$, allowing us to extract these vectors by using the $\bar{x}$ definition (Eq. 4.2a). We therefore have,

$$
\widetilde{\bar{X}}\left(I_{\lambda \lambda_{4}, \mu_{4}}^{m}\right) \bar{x}=\frac{1}{2} \sum_{S_{3} S_{4}}\left[I_{\lambda_{4}, \mu_{2}}^{X_{m}}+\tilde{I}_{\lambda_{4}^{m}, \mu} X_{2}^{m}\right]
$$

Following the above procedure, $Q_{4}$ becomes

$$
Q_{4}=\tilde{x} \tilde{\bar{x}}\left(I_{\lambda_{4}}^{m}, \mu_{4}^{m}\right) \bar{x} x
$$

or, using the definition for $U_{m}$ (Eq. 4.4),

$$
Q_{4}=\tilde{U}_{2}\left(I_{\lambda_{4}, \mu_{4}^{m}}^{m}\right) U_{2}
$$

and the matrix $I_{\lambda 4, \mu 4}^{m}$ has dimensions $\left(L^{2} \times L^{2}\right)$ in the original scalar quantities of $(4.1)$.

The procedures outlined above lead to straightforward generalization for arbitrary products; it is only necessary to separate the odd from the even products, since they result in slightly modified forms.

Even products: We may consider all even products of (4.1) by letting $m=2 \ell$. Following the procedures of quadratic and quartic products, we find that

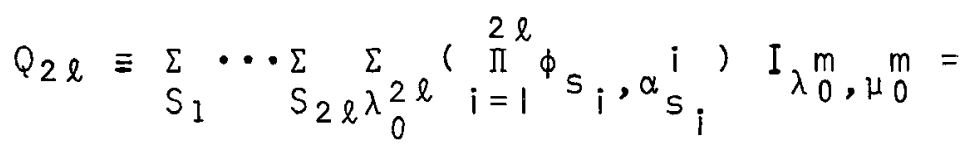

$$
\begin{aligned}
& =\tilde{U}_{\ell}\left(I_{\lambda}{ }_{2}^{m}, \mu_{2 \ell}^{m}\right) U_{\ell}
\end{aligned}
$$

where $I_{\lambda} m_{2 \ell, \mu}^{m} m_{\ell}$ is a symmetric matrix of dimensions $\left(L^{\ell} \times L^{\ell}\right)$ in the scalar quantities $I_{\lambda}^{m}, \mu_{0}^{m}$.

Odd products: For odd products it is only necessary to apply the technique for even products and then use the method for cubic products. Letting $m=2 \ell+1$, 


$$
\begin{aligned}
Q_{2 \ell+1} & \equiv S_{2 \ell+1}^{\sum_{2 \ell} \sum_{2 \ell+1} Q_{2 \ell} S_{2 \ell+1}, \alpha_{S_{2 \ell+1}^{2 \ell+1}}}= \\
& =\tilde{K} \tilde{U}_{\ell}\left(I_{\lambda}^{m}{ }_{2 \ell+1}^{m}, \mu_{2 \ell+1}^{m}\right) \bar{U}_{\ell} X \cdot
\end{aligned}
$$

and $I_{\lambda}^{m} m, \mu_{2 \ell+1}^{m} \quad$ is a symmetric matrix of dimensions $\left(L^{\ell+1} \times L^{\ell+1}\right)$ in the variables $I_{\lambda \mu_{0}, H_{0}^{m}}$.

We may now combine the results of this section (4.27 and 4.28 ) to give a general matrix expression for the non inear terms of $(1.1)$ as represented by (4.1) which becomes,

$$
\begin{aligned}
& B\left(k, \gamma_{k}\right)=\sum_{m=1}^{M}\left[\tilde{U}_{m}\left(I_{\lambda} 2 m, \mu_{2 m}^{2 m}\right) U_{m}+\right. \\
& +\tilde{K} \tilde{U}_{m-1}\left(I_{\lambda}^{2 m-1} 2, \mu_{2 m-1}^{2 m-1}\right) \bar{U}_{m-1}^{2 m-1} \\
& \left.U_{1}\right]
\end{aligned}
$$

If the reduction of the left-hand side of (1.1) requires the summation of all the elements $B\left(k, \gamma_{k}\right)$ for each variable $\left(\phi_{j, \alpha_{j}}\right)$, the summation may be performed on the interaction matrices "I" before time extrapolation begins as is seen from $(3.12)$ and $(4.29)$ as follows:

$$
\begin{aligned}
& \sum_{j} \sum_{j} V_{j, \alpha_{j}}, k, \gamma_{k} B\left(j, \alpha_{j}\right)=\sum_{m=1}^{M}\left[\tilde{U}_{m} I I m U_{m}+\right. \\
& \left.+\tilde{K} \tilde{U}_{m-1} I_{2 m-1} \bar{U}_{m-1} U_{1}\right] \\
& \operatorname{II}_{2 m}\left(k, \gamma_{k}\right) \equiv \sum_{j} \sum_{\alpha_{j}} V_{j, \alpha_{j}}, k, \gamma_{k} I_{2 m} 2 m, \mu_{2 m}^{2 m}\left(j, \alpha_{j}\right)
\end{aligned}
$$

It is important to note that the general nonlinear product can be represented as a quadratic form for even nonlinearity and a similar representation for odd products. The matrices 
$U_{m}$ can be calculated from their definition at any time, and the matrices "I" are known (and remain constant) for all time once the basic equation and the space and time truncation have been established. We shall show subsequently, how (4.29) may be calculated in a straightforward manner. Moreover, a program for performing such calculations on a high-speed digital computer has been developed.

\section{Example 1: Finite difference method}

For a demonstration of the finite-difference method representation of $(1.1)$, let us consider the set of equations representing atmospheric flow and sometimes referred to as the "primitive equations." We shall assume a representation in cartesian coordinates $(x, y, z)$ with the horizontal motion in a tangent plane (a plane tangent to the earth at some latitude) and vertical motion normal to this plane. The physical system will have no external heating (adiabatic) and the usual--and generally highly acceptable--hydrostatic approximation shall not be made. The basic system may be written,

$$
\begin{aligned}
& \frac{\partial \bar{V}}{\partial t}=-\bar{V} \cdot \nabla \bar{V}-2 \Omega \times \bar{V}-\alpha \nabla p-g \bar{k} \\
& \frac{\partial \alpha}{\partial t}=-\bar{V} \cdot \nabla \alpha+\alpha \nabla \cdot \bar{V} \\
& \frac{\partial p}{\partial t}=-\bar{V} \cdot \nabla p+\frac{p}{k-T} \nabla \cdot \bar{V}
\end{aligned}
$$

Here $\bar{V}$ represents the three-dimensional wind vector with components $(u, v, w)$ in the three cartesian directions expressed by the unit vectors $(\bar{i}, \bar{j}, \bar{k})$. s is the rotation vector of the earth with constant magnitude $\Omega$ and components $(0,2 \Omega \cos \theta, 2 \Omega \sin \theta)$, where $\theta$ is the latitude of tangency, $P$ and $\alpha$ are the pressure and density, respectively, $g$ is the constant acceleration of gravity, and $k$ is Poisson's constant. 
In this system there are five independent variables, $N=5$, and they may be represented by $\Phi_{j}$ as follows:

$$
\begin{aligned}
\Phi_{j} & =(u, v, w, \alpha, p) \\
j & =(1,2,3,4,5)
\end{aligned}
$$

$1+$ is immediately apparent that no reduction of the I.h.s. of this system of equations is necessary as we may see by writing the operators defined by $(2.1)$,

$$
\begin{aligned}
& T_{k, j, i}=T_{k, j, 1}=\frac{\partial}{\partial t} \\
& G_{k, j, i}=G_{k, j, 1}=\delta_{k, j}
\end{aligned}
$$

Scrutiny of the right-hand side of (5.1) shows that $M=2$; i.e., we have both linear and quadratic terms. The operators $F_{\lambda}^{m}, m, i, k$ for this system are listed in Table I. If more than one set of operators appears in the table, all the sets must be applied to the dependent variables and the results added. This condition represents the multiple products of operators involving the same variables as discussed following (1.2). If we moreover add the inhomogeneous term $g$, Table I together with (5.2) and (5.3) may be used to give the representation of system (5.1) in the form (1.1).

We now show how this example may be expressed by substituting finite-difference operators for space differentials. As pointed out in section 2, we must establish a grid of points at which the dependent variables are to be evaluated. For simplicity, we shall assume that all the dependent variables are known at the same set of points. Furthermore, the separation between points will be the same in each horizontal layer, and these layers will be equally spaced. Thus an arbitrary point with coordinates ( $x, y, z)$ will be found in the finite-difference net at, 
Table

Space Operators for Finite-Difference Example

$$
F_{\mu}^{2}, 2, i, k \quad(m=2, i=1,2)
$$

\begin{tabular}{|c|c|c|c|c|c|c|c|c|c|c|}
\hline \multirow{2}{*}{$\begin{array}{c}\mathrm{k}= \\
\mu_{0}^{2}\end{array}$} & \multicolumn{2}{|c|}{1} & \multicolumn{2}{|c|}{2} & \multicolumn{2}{|c|}{3} & \multicolumn{2}{|c|}{4} & \multicolumn{2}{|r|}{5} \\
\hline & $i=1$ & $i=2$ & $i=1$ & $i=2$ & $i=1$ & $i=2$ & $i=1$ & $i=2$ & $i=1$ & $i=2$ \\
\hline$(1,1)$ & -1 & $\partial / \partial x$ & 0 & 0 & 0 & 0 & 0 & 0 & 0 & 0 \\
\hline$(1,2)$ & $\partial / \partial y$ & -1 & -1 & $\partial / \partial x$ & 0 & 0 & 0 & 0 & 0 & 0 \\
\hline$(1,3)$ & $\partial / \partial z$ & -1 & 0 & 0 & -1 & $\partial / \partial x$ & 0 & 0 & 0 & 0 \\
\hline$(1,4)$ & 0 & 0 & 0 & 0 & 0 & 0 & $\left\{\begin{array}{l}-1 \\
\partial / \partial x\end{array}\right.$ & $\left\{\begin{array}{c}\partial / \partial x \\
1\end{array}\right.$ & 0 & 0 \\
\hline$(1,5)$ & 0 & 0 & 0 & 0 & 0 & 0 & 0 & 0 & $\left\{\begin{array}{l}\partial / \partial x \\
x\end{array}\right.$ & $\left\{\begin{array}{l}d / d x \\
1 / k-1\end{array}\right.$ \\
\hline$(2,2)$ & 0 & 0 & -1 & $\partial / \partial y$ & 0 & 0 & 0 & 0 & 0 & 0 \\
\hline$(2,3)$ & 0 & 0 & $\partial / \partial z$ & -1 & -1 & $\partial / \partial y$ & 0 & 0 & 0 & 0 \\
\hline$(2,4)$ & 0 & 0 & 0 & 0 & 0 & 0 & $\left\{\begin{array}{l}-1 \\
\partial / \partial y\end{array}\right.$ & $\left\{\begin{array}{c}\partial / \partial y \\
1\end{array}\right.$ & 0 & $\begin{array}{c}0 \\
2\end{array}$ \\
\hline$(2,5)$ & 0 & 0 & 0 & 0 & 0 & 0 & 0 & 0 & $\left\{\begin{array}{l}-1 \\
\partial / \partial y\end{array}\right.$ & $\left\{\begin{array}{l}d / \partial y \\
1 / k-1\end{array}\right.$ \\
\hline$(3,3)$ & 0 & 0 & 0 & 0 & -1 & $\partial / \partial z$ & $\begin{array}{l}0 \\
1\end{array}$ & $\begin{array}{c}0 \\
27\end{array}$ & 0 & 0 \\
\hline$(3,4)$ & 0 & 0 & 0 & 0 & 0 & 0 & $\left\{\begin{array}{l}-1 \\
\partial z\end{array}\right.$ & $\left\{\begin{array}{c}0 / 0 \\
1\end{array}\right.$ & $\begin{array}{r}0 \\
-1\end{array}$ & $\begin{array}{c}0 \\
\partial / \partial z\end{array}$ \\
\hline$(3,5)$ & 0 & 0 & 0 & 0 & 0 & 0 & 0 & 0 & $\{\partial / \partial z$ & $\{1 / k-1$ \\
\hline$(4,4)$ & 0 & 0 & 0 & 0 & 0 & 0 & 0 & 0 & 0 & 0 \\
\hline$(4,5)$ & -1 & $\partial / \partial x$ & -1 & $\partial / \partial y$ & -1 & $\partial / \partial z$ & 0 & 0 & 0 & 0 \\
\hline$(5,5)$ & 0 & 0 & 0 & 0 & 0 & 0 & 0 & 0 & 0 & 0 \\
\hline
\end{tabular}




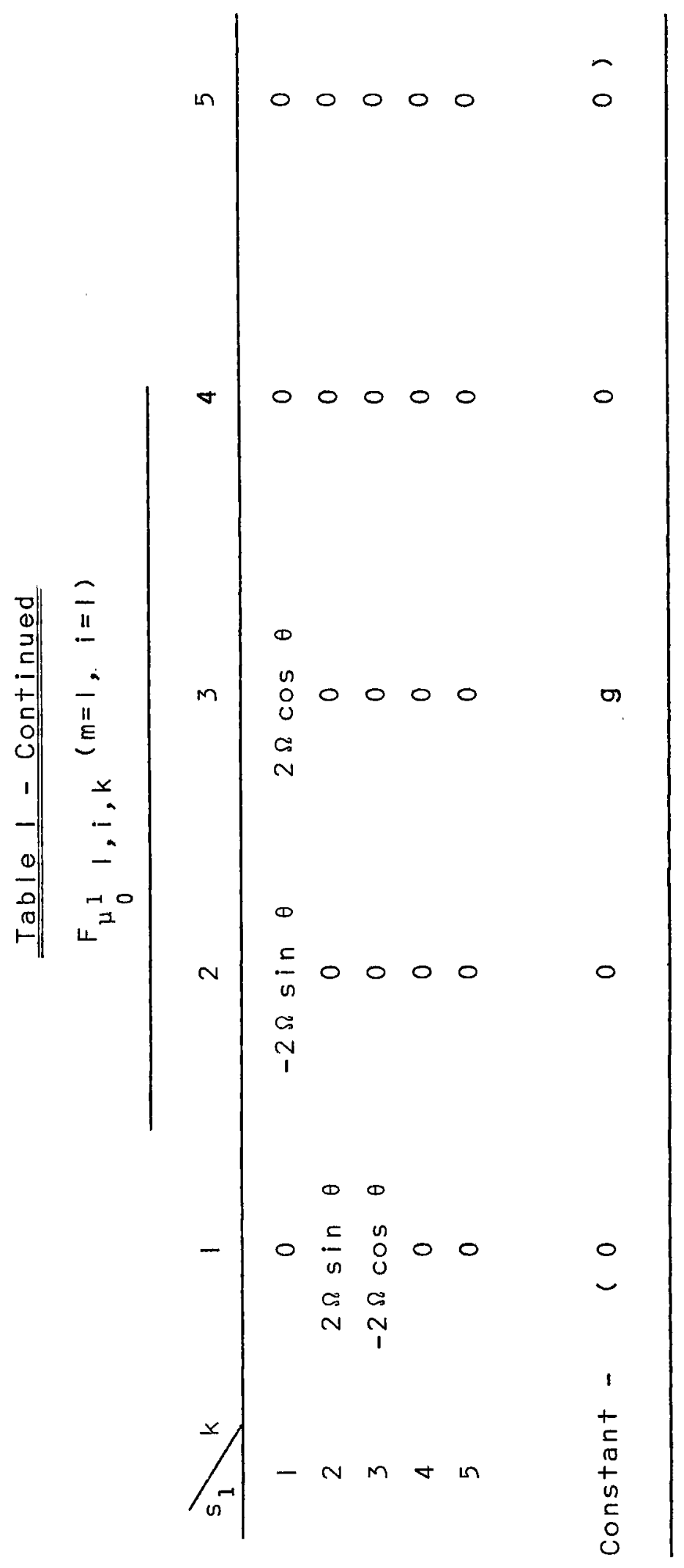




$$
\begin{aligned}
& x=p \Delta x=p \Delta s ; y=q \Delta y=q \Delta s ; z=r \Delta z \\
& 0 \leq p \leq p-1 ; 0 \leq q \leq Q-1 ; 0 \leq r \leq R-1
\end{aligned}
$$

or we may refer to the location in terms of the indices $\left(x_{0}, y_{0}, z_{0}\right) \rightarrow(p, q, r)$. Since we wish to identify each point by a single index (the number $\gamma$ ), we define $(p, q, r$ ) by the relation

$$
r(p, q, r)=P Q r+P q+p+1
$$

Thus each point is uniquely indexed and the total number of points will be PQR. The value of any variable at the point $\gamma$ will be $\phi_{j, \gamma_{j}}$, where we include the subscript on $\gamma$ to indicate the dependent variable to which reference is made. Since there are $N=5$ dependent variable functions, the total number of discrete variable quantities is $L=5 P Q R$ (see 2.3).

The finite-difference representation of the non-linear terms follows the procedure outlined in section 2. Let us select as a representative example the two entries for $\mu_{0}^{2}=(1,4), k=4$ from Table 1 . From the Table, $(1,1)$ and $(2.5)$,

$$
\begin{aligned}
& F_{\mu_{0}^{2}, 2,1,4}\left(\Phi_{1}\right) F_{\mu_{0}^{2}, 2,2,4}\left(\Phi_{4}\right)=-\Phi_{1} \frac{\partial \Phi_{4}}{\partial x}+\Phi_{4} \frac{\partial \Phi_{1}}{\partial x}=
\end{aligned}
$$

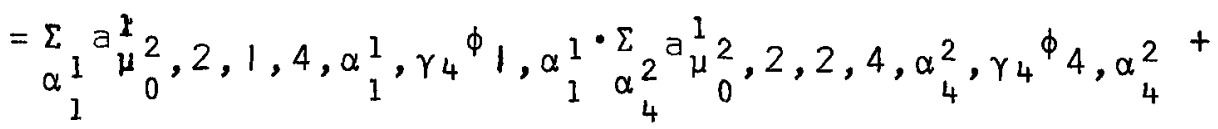

$$
\begin{aligned}
& +\sum_{\alpha}{ }_{1} a_{\mu}^{2}{ }_{0}^{2}, 2,1,4, \alpha_{1}^{1}, \gamma_{4} \phi_{1}, \alpha_{1}^{1} \sum_{\alpha_{4}^{2}} a_{\mu}^{2} \mu_{0}^{2}, 2,2,4, \alpha_{4}^{2}, \gamma_{4} \phi_{4}, \alpha_{4}^{2}= \\
& =\sum_{\lambda}^{2} I_{0} \lambda_{0}^{2}, \mu_{0}^{2\left(4, \gamma_{4}\right) \phi} 1, \alpha_{1}^{1 \phi} 4, \alpha_{4}^{2} \\
& \lambda_{0}^{2}=\left(\alpha_{1}^{1}, \alpha_{4}^{2}\right) ; \mu_{0}^{2}=(1,4)
\end{aligned}
$$


where we have used the superscript on the a's because more than one product involving the same dependent variables exists. Let $\gamma_{4}$ represent an arbitrary (but not boundary) point in the net defined above with index values $(p, q, r)$. We shall use the centered finite-difference operator to approximate the differential operator

$$
\left.\frac{\partial \Phi}{\partial x}\right|_{\gamma_{4}} \approx \frac{1}{2 \Delta x}\left[\phi_{i, \gamma_{4}+1}-\phi_{i, \gamma_{4}-1}\right] \text {, }
$$

an approximation commonly used and adequate to exemplify our procedure. Using ( 5.6$)$, the coefficients of (5.5) may be written in Kronecker delta notation,

$$
\begin{aligned}
& a_{0}^{1} 2,2,1,4, \alpha_{1}^{1}, \gamma_{4}=-\delta_{\alpha_{1}^{1}, \gamma_{4}}^{2} \\
& a_{0}^{1} 2,2,2,4, \alpha_{4}^{2}, \gamma_{4}=\frac{1}{2 \Delta x}\left[\delta_{\alpha_{4}^{2}, \gamma_{4}+1}-\delta_{\alpha_{4}^{2}, \gamma_{4}-1}\right] \\
& a_{\mu_{0}^{2}, 2,1,4, \alpha_{1}^{1}, \gamma_{4}}^{2}=\frac{1}{2 \Delta x}\left[\delta_{\alpha_{1}^{1}, \gamma_{4}+1}-\delta_{\alpha_{1}^{1}, \gamma_{4}-1}\right] \\
& a_{\mu}^{2}, 2,2,4, \alpha_{4}^{2}, \gamma_{4}=\delta_{\alpha_{4}^{2}, \gamma_{4}}
\end{aligned}
$$

Although these fields of coefficients have PQR elements, we see that most are zero. These values then yield for the interaction coefficients,

$$
\begin{gathered}
I_{\mu_{0}, \mu_{0}^{2}}\left(4, \gamma_{4}\right)=\frac{1}{2 \Delta x}\left[\delta_{\alpha_{4}, \gamma_{4}}^{2}\left(\delta_{\alpha_{1}^{1}, \gamma_{4}+1}-\delta_{\alpha_{1}^{1}, \gamma_{4}+1}\right)\right. \\
\left.-\delta_{\alpha_{1}^{1}, \gamma_{4}}\left(\delta_{\alpha_{4}^{2}, \gamma_{4}+1}-\delta_{\alpha_{4}^{2}, \gamma_{4}-1}\right)\right]
\end{gathered}
$$

a field of $(P Q R)^{2}$ quantities, again mostly zero. We shall see later that the zero elements can be eliminated from actual calculation. There exists a set of interaction coefficients for 
each element in Table l and for each point ( $k$ ) in the grid. Care must be taken at the boundaries, but we assume that appropriate boundary conditions are specified and that corresponding finite-difference operators can be unambiguously specified.

The interaction coefficients (5.8) can be listed in matrix form by the procedure outlined in section 4, which needs no further elaboration. The final matrix equation for this system becomes,

$$
\begin{aligned}
& \frac{\partial \phi_{k, \gamma_{k}}}{\partial t}=-g \delta_{k, 3}+\tilde{\dot{K}}\left\{I_{\lambda \lambda_{1}^{1}, \mu_{1}^{1}}\left(k, \gamma_{k}\right)\right\} x+\tilde{x}\left\{I_{\lambda_{2}^{2}, \mu_{2}^{2}}\left(k, \gamma_{k}\right)\right\}_{x} \\
& x=\left\{\phi_{j, \alpha_{j}}\right\}
\end{aligned}
$$

where the matrices $I_{\lambda{ }_{1}^{1}, \mu} \frac{1}{1}$ are derived from application of the operators $F_{\mu} 1,1,1, k$ of Table 1 and the matrices $I_{\lambda_{2}^{2}, \mu_{2}^{2}}$ arise from the application of the operators $F_{\mu}^{2}, 2,1, k$ and contain elements of the type described by (5.8).

\section{Example 2: Spectral method}

For an example involving the spectral approach we shall use a somewhat simpler physical model--applying more approximations--than the one used in the previous section, but which nevertheless incorporates coupling of terms involving the time operators so that the results of Section 3 may be utilized. The model is generally referred to as a "General Circulation Model" and is very similar to one described by Phillips (1956).

The flow field in this model is described by its rotational part (the vorticity) by use of the quasi-geostrophic and quasi-hydrostatic approximations. The dependent variables are described in pressure coordinates with spherical surfaces respresenting constant pressure surfaces having coordinates 
$\lambda$ (longitude) and $\mu$ (sin of latitude). (Note: The coordinate notation $(\lambda, \mu)$ is used here to conform to convention. These variables have no relation to the indices used elsewhere in this paper, and since all operations are defined, no confusion should ensue.) The vertical velocity (in pressure) is specified at the top and bottom of the atmosphere. Friction is incorporated at the surface proportional to the vorticity and non-adiabatic heating is included proportional to the mean temperature of the atmosphere.* To simplify further, the pressure dependence is approximated by evaluating the dependent variables at two levels only (finitedifference approximation in the vertical in which the pressure interval is one-half the pressure depth of the atmosphere), and the dependent variables used are the stream functions at these two levels, derivable from the geostrophic vorticity. The equations for this system are **

$$
\begin{aligned}
\frac{\partial}{\partial \dagger}\left[\nabla^{2} \psi_{1}-h^{2}\left(\psi_{1}-\psi_{3}\right)\right] & =-2 \frac{\partial \psi_{1}}{\partial \lambda}+\frac{\partial\left(\nabla^{2} \psi_{1}+h^{2} \psi_{3}, \psi_{1}\right)}{\partial(\lambda, \mu)}-k_{1}\left(\psi_{3}-\psi_{1}\right) \\
\frac{\partial}{\partial t}\left[\nabla^{2} \psi_{3}+h^{2}\left(\psi_{1}-\psi_{3}\right)\right] & =-2 \frac{\partial \psi_{3}}{\partial \lambda}+\frac{\partial\left(\nabla^{2} \psi_{3}+h^{2} \psi_{1}, \psi_{3}\right)}{\partial(\lambda, \mu)}+k_{1}\left(\psi_{3}-\psi_{1}\right) \\
+ & k_{2}\left(\nabla^{2} \psi_{1}-3 \nabla^{2} \psi_{3}\right)
\end{aligned}
$$

The parameters $\psi_{1}, \psi_{3}$ (the dependent variables) are the stream functions at the two levels and are functions of $(t$, $\lambda, \mu)$. The quantities $h^{2}, K_{1}, K_{2}$ are constants and depend on the character of friction, heating and static stability (also assumed constant). The Laplacian operator is taken in a spherical surface. point.

* The model differs from that of Phillips (1956) on this

**This model in spectral form is currently being investigated for its predictive characteristics with support from the National Science Foundation, Grant GA-76I. 
We now represent system (6.1) in the notation of (1.1). Since there are only two dependent variables, $N=2$ and

$$
\left(\Phi_{1}, \Phi_{2}\right)=\left(\psi_{1}, \psi_{3}\right)
$$

For the time-space operators defined by (2.1),

$$
\begin{aligned}
T_{k, j, i} & =T_{k, j, 1}=\frac{\partial}{\partial t} \\
G_{k, j, i} & =G_{k, j, 1} \equiv G_{k, j} \\
I & =1 .
\end{aligned}
$$

The operators $G_{k, j}$ are listed in Table 2 . From (6.1) we see that $M=2$; the operators $F_{\mu_{0}}, m, i, k$ are listed in Table 3. With these definitions, (6.1) may be expressed as $(1.1)$.

The dependent variables $\Phi_{j}$ are now expanded in terms of orthogonal polynomials in $(\lambda, \mu)$ where we use surface spherical harmonics (see Hobson, 1955),

$$
P_{\alpha_{j}}(\gamma, \mu)=Y_{\alpha_{j}}(\lambda, \mu)=\exp \left(i \ell_{\alpha_{j}} \lambda\right) P_{\alpha_{j}}(\mu)
$$

with the properties

$$
\begin{gathered}
\nabla^{2} Y_{\alpha_{j}}=-c_{\alpha_{j}}^{2}{ }^{Y} \alpha_{j} \\
\frac{\partial}{\partial \lambda} Y_{\alpha_{j}}=i \ell_{\alpha_{j}}{ }^{\alpha_{\alpha_{j}}} \\
\delta Y_{\alpha_{j}} Y_{\alpha_{k}^{*}} d \lambda d \mu=\delta_{\alpha_{j}, \alpha_{k}}
\end{gathered}
$$

where $c_{\alpha_{j}}^{2}$ and $\ell_{\alpha_{j}}$ are constants. Thus we have established the set of time-dependent variables $\phi_{1, \alpha_{1}}$ and $\phi_{2, \alpha_{2}}$. 
Table 2

operators $G_{k, j}$ for Spectral Model

\begin{tabular}{ccc}
\hline & 1 & 2 \\
\hline & $\nabla^{2}-h^{2}$ & $h^{2}$ \\
\hline
\end{tabular}

Table 3

Operators $F_{\mu_{0}^{m}, m, i, k}$ for Spectral Model

$$
F_{0} \frac{1}{0}, 1,1, k \quad(m=1, i=1)
$$

\begin{tabular}{lcc}
$s_{1} k_{1}^{k}$ & 1 & 2 \\
\hline$k_{1}-2 \partial / \partial \lambda$ & $k_{2} \nabla^{2}-k_{1}$ \\
$-k_{1}$ & $k_{1}-2 \partial / \partial \lambda-3 K_{2} \nabla^{2}$
\end{tabular}

$$
\mathrm{F}_{\mu_{0}^{2}, 2, i, k}(m=2, i=1,2)
$$

\begin{tabular}{|c|c|c|c|c|}
\hline$k=$ & & & 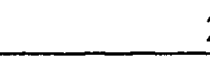 & \\
\hline$\mu_{0}^{2}$ & $i=1$ & $i=2$ & $i=1$ & $i=2$ \\
\hline$(1,1)$ & $\left\{\begin{array}{r}\nabla^{2} \partial / \partial \lambda \\
-\nabla^{2} \partial / \partial \mu\end{array}\right.$ & $\left\{\begin{array}{l}\partial / \partial \mu \\
\partial / \partial \lambda\end{array}\right.$ & 0 & 0 \\
\hline$(1,2)$ & $\left\{\begin{array}{r}\partial / \partial \mu \\
-\partial / \partial \lambda\end{array}\right.$ & $\left\{\begin{array}{l}h^{2} \partial / \partial \lambda \\
h^{2} \partial / \partial \mu\end{array}\right.$ & $\left\{\begin{array}{r}h^{2} \partial / \partial \lambda \\
-h^{2} \partial / \partial \mu\end{array}\right.$ & $\left\{\begin{array}{l}\partial / \partial \mu \\
\partial / \partial \lambda\end{array}\right.$ \\
\hline$(2,2)$ & 0 & 0 & $\left\{\begin{array}{r}\nabla^{2} \partial / \partial \lambda \\
-\nabla^{2} \partial / \partial \mu\end{array}\right.$ & $\left\{\begin{array}{l}\partial / \partial \mu \\
\partial / \partial \lambda\end{array}\right.$ \\
\hline
\end{tabular}


$1 t$ is not necessary to specify numerically the series limit, and we shall therefore keep the range as $1 \leq \alpha_{j} \leq n_{j}$; we then have $L=n_{1}+n_{2}$. Using (6.4), (6.5) and Table 2, we may represent the $a_{k, j, \alpha_{j}, \gamma_{k}, i}$ for this model from equation (2.10); this yields as an example for $k=1, j=1$,

$$
\begin{aligned}
a_{1,1, \alpha_{1}, \gamma_{1}} & =\delta G_{1,1}\left(Y_{\alpha_{1}}\right) Y_{\gamma_{1}}^{*} \delta \lambda \delta \mu \\
& =\delta\left(-C_{\alpha_{1}}^{2}-h^{2}\right) Y_{\alpha_{1}} Y_{\gamma_{1}}^{*} d \lambda d \mu \\
& =-\left(C_{\alpha_{1}}^{2}+h^{2}\right) \delta_{\alpha_{1}, \gamma_{1}} .
\end{aligned}
$$

For the terms involving the $F$ operators (Table 3 ), using $(2.12),(6.4)$ and (6.5), we have as an example with $\mu_{0}^{2}=$ $(1,2), \lambda_{0}^{2}=\left(\alpha_{1}^{1}, \alpha_{2}^{2}\right)$,

$$
\begin{aligned}
& \int F_{\mu_{0}^{2}, 2,1,1}\left(\Phi_{1}\right) F_{\mu_{0}^{2}, 2,2,1}\left(\Phi_{2}\right) Y_{Y_{2}}^{*} d \lambda d \mu \\
& =\int\left[h^{2} \frac{\partial \Phi_{1}}{\partial \mu} \frac{\partial \Phi_{2}}{\partial \lambda}-h^{2} \frac{\partial \Phi_{1}}{\partial \lambda} \frac{\partial \Phi_{2}}{\partial \mu}\right] Y_{\gamma_{1}^{*}}^{*} d \lambda d \mu \\
& =\quad \sum_{\lambda} \phi_{0} 1, \alpha_{1}^{1} \Phi_{2, \alpha_{2}^{2}} I_{\lambda_{0}^{2}, \mu_{0}^{2}}\left(I, \gamma_{1}\right)
\end{aligned}
$$

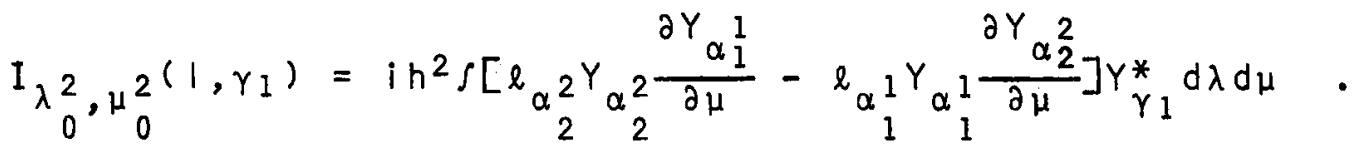

All the applicable terms may be calculated as the above examples and need not be listed here in detail. The functions $B_{k, \gamma_{k}}$ (Eq. 3.1) are known from evaluation of the type (6.7).

The terms involving variation in time may be uncoupled following the procedures outlined in section 3. In this example it is not necessary to establish the method of time truncation to perform the uncoupling. Following section 3 and suppressing the index $i$ since $I=1$, 


$$
x=\left(\begin{array}{c}
\left\{\phi_{1}, \alpha_{1}^{1}\right\} \\
\left\{\phi_{2, \alpha_{2}^{2}}\right\}
\end{array}\right)
$$

From (6.3),

$$
\bar{T}_{k, j}=\frac{\partial}{\partial t} I_{j} ; \quad T_{k}=\frac{\partial}{\partial t}\left(\begin{array}{ll}
I_{1} & 0 \\
0 & I_{2}
\end{array}\right)
$$

From (3.5) and $(6.6)$,

$$
\begin{aligned}
& \text { । } \cdots \quad \gamma_{1} \quad \cdots n_{1} \quad n_{1}+1 \cdots n_{1}+\gamma_{1} \cdots n_{1}+n_{2} \\
& \tilde{A}_{1, \gamma_{1}}=\left[\begin{array}{lllllllllll}
0 & \cdots & -C_{\gamma_{1}}^{2}-h^{2} & \cdots & 0 & 0 & \cdots & h^{2} & \cdots & 0
\end{array}\right]
\end{aligned}
$$

etc. Using $(6.9)$ and $(6.10)$,

$$
\begin{aligned}
& T_{k, \gamma_{k}}=\tilde{A}_{k, \gamma_{k}} T_{k}=\frac{\partial}{\partial t} \tilde{A}_{k, \gamma_{k}} \\
& T=\frac{\partial}{\partial t} A ; A=\left\{\tilde{A}_{k, \gamma_{k}}\right\} .
\end{aligned}
$$

Noting the values of the vectors $A_{k, \gamma_{k}}$ as described by the example (6.10), we may spell out the matrix $A$ as follows assuming $n_{2}>n_{1}$,

$$
A=
$$

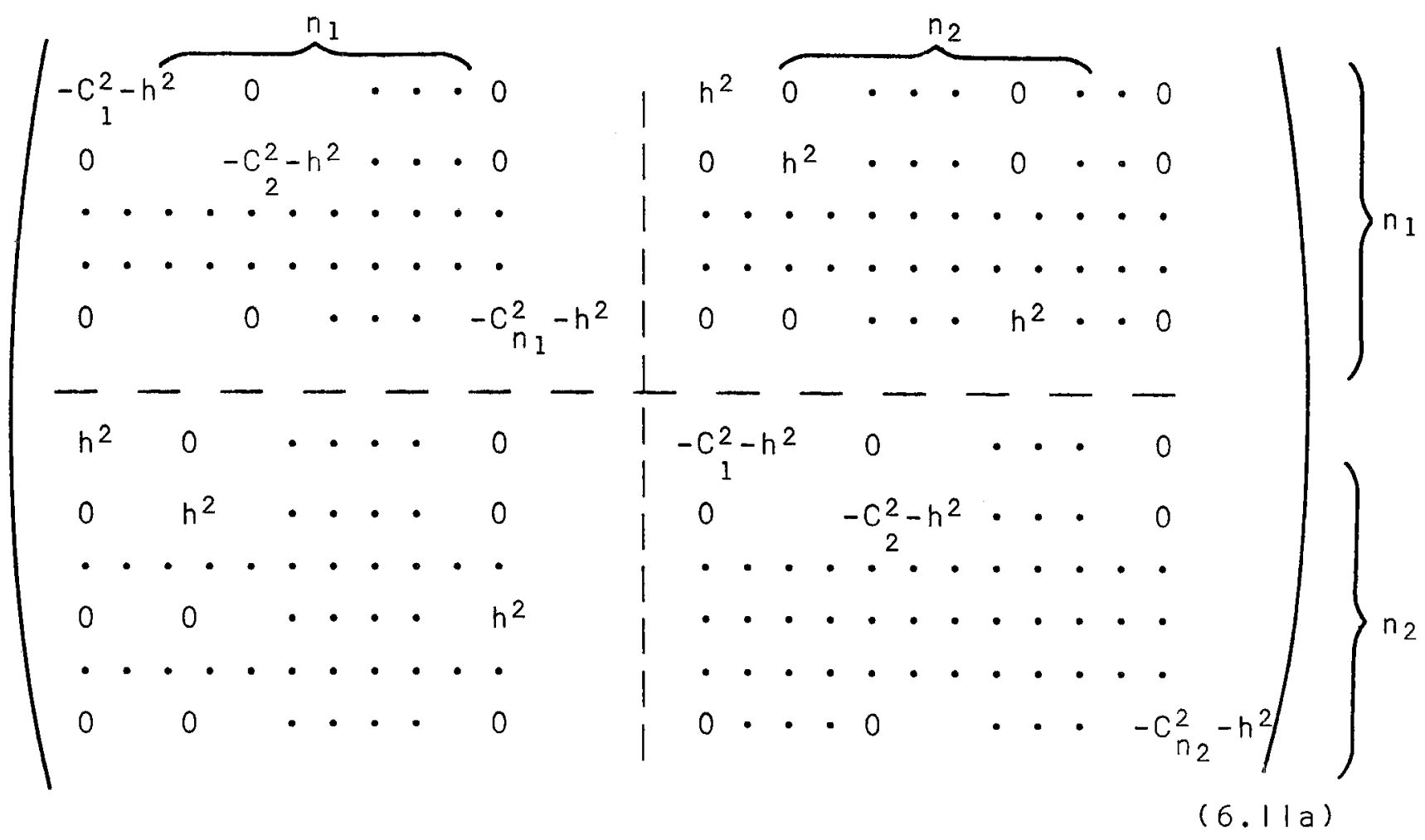


At this point we deviate from the development of section 3 because we may write immediately,

$$
A \frac{\partial X}{\partial t}=B
$$

where

$$
B=\left\{B_{k, \gamma_{k}}\right\}
$$

the latter vector elements having been evaluated in the discussion above up to and including (6.7). Now assuming that A has an inverse* with elements $v_{j, \alpha_{j}, k, \gamma_{k}}$, the time differential equation for each dependent variable may be written,

$$
\frac{\partial}{\partial t} \phi_{k, \gamma_{k}}=\sum_{j=1}^{2} \sum_{j} v_{j, \alpha}, k, \gamma_{k} B_{j, \alpha}
$$

the uncoupled equation comparable to (3.12). The complete expression for $(1.1)$ in matrix form for this example (6.1) can now be written as

$$
\frac{\partial \phi_{k}, \gamma_{k}}{\partial t}=\tilde{K}\left\{I I_{1}\left(k, \gamma_{k}\right)\right\} x+\tilde{x}\left\{I I_{2}\left(k, \gamma_{k}\right)\right\} x
$$

where the matrices II are defined by (4.30) using the elements $\checkmark$ from the inverse of $A$ (6.11a) and the matrices I from the elements of Table 2 developed as in (6.7). Any truncation scheme may now be used to solve (6.15) in time, provided the initial conditions are properly specified.

7. Calculation procedure

We have seen how it is possible to reduce a general, nonlinear differential equation (1.1) to the form (3.12) for numerical calculation. We furthermore note that all the

* If a degeneracy were to exist in the system, it could be removed by reducing the number of dependent variables. 
quantities on the right-hand side of (3.12) are known at any time $t=p \Delta t$. Since there are $L$ (Eq. 2.3) dependent variables, there are $L$ equations of the type (3.12) which must be solved at each time step. The linear terms offer no computational complexities since the coefficient fields

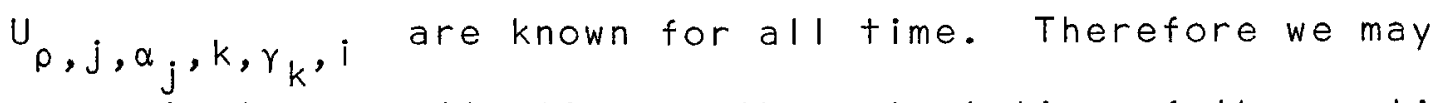
concentrate our attention on the calculation of the nonlinear terms.

The nonlinear terms may be represented--regardless of the order of nonlinearity--by a bilinear form (quadratic in the case of even products) as seen from (4.30). If we represent an arbitrary bilinear form as

$$
N L_{\ell} \equiv D_{\ell, 1} H_{\ell} D_{\ell, 2}
$$

where $1 \leq \ell \leq 2 M$, we see that vectors $D_{\ell, 1}$ and $D_{\ell, 2}$ and the matrices $H_{2}$ are defined for odd and even products from (4.30) in Table 4 .

Table 4

Vectors and Matrices of the

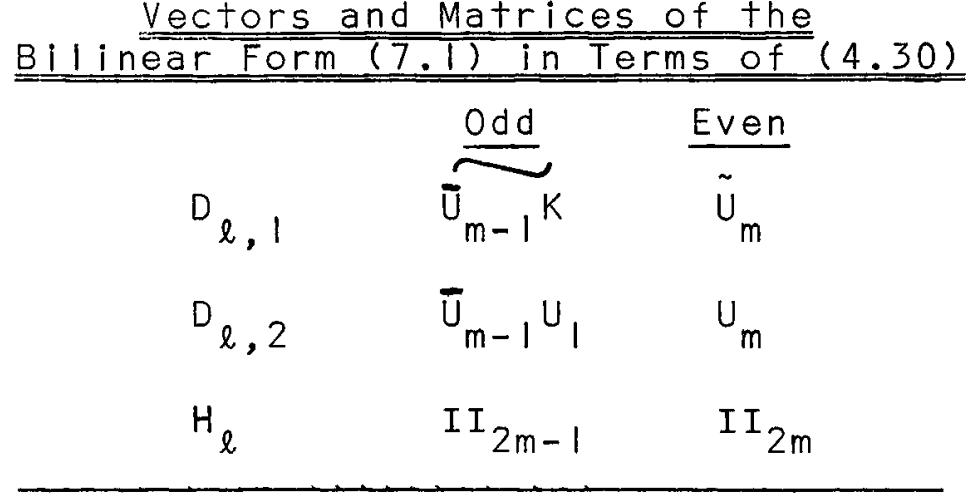

The vectors $D_{\ell, 1}$ and $D_{\ell, 2}$ may be calculated from the values of the vector $x(p \Delta t)--$ known quantities--by usual matrix multiplication procedures as implied by (4.2), (4.3) and (4.4). since all the products are of the form (7.1), we may delete the l subscript and describe the calculation of any one of the bilinear forms, $\mathrm{NL}_{\ell}$. We note, however, that for each 
equation of the type (3.12)--and there are $L$ of them-there may exist a bilinear product of the form (7.1); thus we refer to the product for a given equation as $N L_{k}(I \leq k \leq L)$, remembering that the degree of nonlinearity of this term is implied (the subscript $\ell$ having been suppressed).

With elements of $D_{1}, D_{2}$, and $H_{k}$ known,

$$
D_{1}=\left[d_{1 i}\right] \quad D_{2}=\left\{d_{2 j}\right\} \quad H_{k}=\left\{h_{i, j, k}\right\},
$$

we may establish the ordered set

$$
a_{r}=(i, j, k, h)_{r}
$$

From this set we may establish the bilinear product for the $k^{\text {th }}$ equation by multiplying the $i^{\text {th }}$ element of $D_{1}$ by the $j^{\text {th }}$ element of $D_{2}$ by the interaction coefficient $h_{i, j, k}$. We now establish the set (not necessarily ordered) of all subsets $a_{r}$,

$$
A=\left\{a_{r}\right\}
$$

This set includes all the information necessary to determine the nonlinear products for a given computation time $\rho \Delta t$, and contains $L^{2 l}$ elements $a_{r}$. We have already seen, especially for the finite-difference method, that many--if not most-- of the $h_{i, j, k}$ vanish. We therefore extract from $A$ the subset of all elements for which $h_{i, j, k}$ does not vanish and define this subset $A^{\prime}$. The information contained in this set ( $A^{\prime}$ ) may now be used to compute the nonlinear products at any time that the elements of $D_{1}$ and $D_{2}$ are known and thus extend the calculation to any desired time according to $(3.12)^{*}$. Certain computational efficiencies may be effected as follows:

* The method described above is extremely well suited to application on digital computers and has been programmed for the model used in section 6 . 
1) We note that for a given order of nonlinearity, say $\ell_{1}$, the vectors $D_{1}$ and $D_{2}$ do not depend on the index $k$; however, the interaction matrix elements do. Thus we should order the set $A^{\prime}$ such that all values of $k$ are incorporated before $i$ and $j$ are changed. We might have,

$$
\begin{aligned}
a_{r} & =\left(i_{1}, j_{1}, k_{1}, h_{1}\right)_{r} \\
a_{r+1} & =\left(i_{1}, j_{1}, k_{1}+1, h_{2}\right)_{r+1} \\
\cdot & \\
a_{r+n} & =\left(i_{1}, j_{1}, k_{1}+n, h_{n+1}\right)_{r+n} \\
a_{r_{1}+n+1} & =\left(i_{2}, j_{2}, k_{1}, h_{n+2}\right)_{r+n+1} \\
e+c . &
\end{aligned}
$$

We need now compute the product $D_{1, i_{1}} D_{2, j_{1}}$ only once and multiply it by the $n+l$ values of $h$, cumulating the results in the partial sums which will make up the new values of the dependent variables $\Phi_{k}$ at $t=(p+1) \Delta t$.

2) If the dependent variables ( $x$ ) which make up the vectors $D_{1}$ and $D_{2}$ are complex, and certain relationships exist between the real and imaginary parts*, the set $A^{\prime}$ may be further ordered on $i$ and $j$ to avoid redundant multiplication.

3) For even products of the nonlinear terms, we see from (4.27) that the bilinear form is a quadratic form. Since the interaction matrices are symmetric, it is only necessary to compute one-half of the matrix product, say above the principal diagonal, and double the values.

* Such conditions might exist so that variables describing physical quantities are real. 
Many variations of ordering of the subsets $a_{r}$ may be made depending on individual requirements. As an example, if storage of elements creates difficulties, the subset ${ }^{a_{r}}$ may be expanded to include the number of variations of $k$ which exist for given $i$ and $j$. Thus $i, j$, and $k$ need be enumerated only once for this set of $a_{r}$, with considerable saving in storage. The possibilities for ordering are many and need not be detailed; they will become apparent when a computation is prepared.

8. Conclusion

It has been shown that a general class of nonlinear partial differential equations in time and space can be represented in a form which may be easily programmed for computation on a digital computer. To prepare any set of equations for computation, a truncation scheme must be selected to represent the dependent variables as a discrete set of numbers; continuous solutions are generally not available. The computational form described is applicable for both the "spectral" and "finite-difference" truncation schemes, two methods most frequently used to reduce the continuous variables to a finite set. The truncation methods have been applied to the space-dependent aspects of the variables only. No specific truncation procedure has been applied to the time dependence of the system although how such truncation might be achieved has been indicated.

In the final computational form, all nonlinear terms are expressed as bilinear forms, the matrices of which are independent of time; i.e., they are not functions of the dependent variables. The vectors of the bilinear form depend only on the dependent variables of the system and are easily calculated at any time that the variables are known. The entire form, it is shown, is readily programmable for computation on a digital computer. The system may furthermore 
be reduced to minimum calculation by deleting all zeromultiplications, thereby making it practicable for the finitedifference method.

Considerable flexibility in the selection of a time truncation scheme is available for the final computational form. Since the space truncation has been represented in the interaction matrices, no confusion between space and time truncation need be encountered. There will undoubtedly be a relationship between these two truncation procedures for computation stability, but this relationship may be investigated directly from the characteristics of the interaction matrices. Furthermore, from the properties of these matrices, some estimates of truncation errors may be established; these errors will be based on truncation procedures which lead to stable computations.

As mentioned earlier, it is not the purpose of this paper to evaluate the relative merits of the finite-difference or spectral methods. Since they can both be represented formally in an identical way, however, it may be possible to use this representation to investigate the differences and similarities of these methods.

Finally, from a practical point of view, the computational form of the general differential equation (1.1) can be programmed for digital computation--at least the nonlinear terms which involve only space truncation--once and for all. Thus each investigator need not prepare a new program for calculation. He need merely specify his time truncation scheme, the order of his vector $x$, necessary initial conditions, the order of nonlinearity $(M)$, and the interaction matrices $I_{m}$ in terms of the non-trivial sets $A^{\prime}{ }_{m}$. Although such a general program has not yet been prepared, the technique has been used satisfactorily for the model discussed in section 6 and for simple models such as the Barotropic Vorticity Equation. 


\section{REFERENCES}

Baer, F., 1961: The extended numerical integration of a single barotropic model. J. Meteor., 18, 319-339.

, 1964: Integration with the spectral vorticity equation. J.Atmos. Sci., 21, 260-276.

Bryan, K., 1959: A numerical investigation of certain features of the general circulation. Tellus, 1 , $163-174$.

Charney, J.G., R. Fjфrtoft, J. von Newman, 1950: Numerical integrations of the barotropic vorticity equation. Tellus, 2, 237-254.

El Isaesser, Hugh W., 1966: Evaluation of spectral versus grid methods of hemispheric numerical weather prediction. J. Appl. Meteor., 5 , 246-262.

Hobson, E. W., 1955: The Theory of Spherical and Ellipsoidal Harmonics. New York, Chelsea Publishing Co., $500 \mathrm{PP}$.

Leith, C., 1965: Numerical simulation of the earth's atmosphere. Methods of Computational Physics, Academic Press, 1-28.

Mintz, Y., 1965: Very long term global integration of the primitive equations of atmospheric motion. W.M.O. Technical Note No. 66, 141-167.

Phillips, N. A., 1956: The general circulation of the atmosphere: a numerical experiment. Quart. J.R. Meteor. Soc., 82, 123-164.

Richardson, L. F., 1922: Weather Prediction by Numerical Process. Cambridge University Press, London, $236 \mathrm{pp}$.

Richtmyer, R. D., 1957: Difference Methods for Initial Value Problems. Interscience Publishers, New York, $238 \mathrm{PP}$.

Silberman, 1., 1954: Planetary waves in the atmosphere. J. Meteor., 11, 27-34.

Smagorinsky, J., S. Manabe, J. L. Holloway, Jr., 1965: Numerical results from a nine-level general circulation model of the atmosphere. Mon. Wea. Rev., 93, 727-768. 